VII. 1 - Phen y l-2-methyl-3.5-bis - s ty ry pyrazoli u m-jodid

Analog I aus $1 \mathrm{~g} 1-\mathrm{Phenyl}-2.3 .5-\mathrm{trimethyl-}$ p y r a o li u m jo did und $2 \mathrm{~g}$ frisch destilliertem B e n z a ldehyd. Elfenbeinfarbene Nädelchen. Schmp. $237^{\circ}$.

$\begin{array}{ll}\mathrm{C}_{26} \mathrm{H}_{23} \mathrm{~N}_{2} \text { J. } & \text { Ber.: C 63,67, H 4,69, N 5,71. } \\ & \text { Gef.: C 63,51, H 4,70, N 5,65. }\end{array}$

VIII. 1 - Pheny $1-2-\mathrm{meth}$ y $\mathrm{l}-3.5-\mathrm{b}$ is methoxystyryl-pyrazolium-jodid

Analog I aus $1 \mathrm{~g} 1-\mathrm{Phenyl}-2.3 .5-\mathrm{trimethyl-}$ pyrazolium jodid und $2 \mathrm{~g} \mathrm{Anis} \mathrm{a} \mathrm{lde} \mathrm{hyd.} \mathrm{Feine}$ blaßgelbe Nädelchen. Schmp. $246^{\circ}$.

$\mathrm{C}_{28} \mathrm{H}_{27} \mathrm{O}_{2} \mathrm{~N}_{2}$ J. Ber.: C 61,09, H 4,09, N 5,71. Gef.: C 61,01, H 4,70, N 5,61.

IX. 1 - Pheny l-2-methy $1-3.5-b$ is - dimet hyla minostyryl-pyrazolium-jodid

Kondensation erfolgt wie bei I mit 1 g 1 - Phenyl2.3.5 - trimethy.l-pyrazoliumjodid und $2 \mathrm{~g}$ Dimethylaminobenzaldehyd. Es wird jedoch 9 Stdn. unter Rückfluß gekocht. Über Nacht kristallisiert das Farbsalz (Jodid) aus.

Durch Lösen in Methanol und Zugabe von wäßriger Natriumperchloratlösung wird das schwerer lösliche P e r - c hlorat erhalten. Beide bilden nach dem Umkristallisieren aus Methanol glänzende orangerote Nädelchen. Schmp. des Jodids $234^{\circ}$, Schmp. des Perchlorats $258^{\circ}$ (Zers.).

$\begin{array}{ll}\mathrm{C}_{30} \mathrm{H}_{33} \mathrm{~N}_{4} \mathrm{~J} . & \text { Ber.: } 62,50 \text {, H 5,72, N 9,72. } \\ & \text { Gef.: } 62,65 \text {, H 5,81, N 9,67. }\end{array}$

X. 1 - Phenyl-2-methyl-5-dimethylaminphenyldivinylen-pyrazolium-jodid

Analog I aus $1 \mathrm{~g} 1$-Phenyl-2.5-dimethylpyrazoliumjodid und 1 g $p$-Dimethyla minozimtaldehyd. Das Farbsalz scheidet sich erst nach dem Einengen der Lösung als clunkelrotbraunes Pulver aus. Nach Umkristallisieren aus Methanol dunkelrotbraune Kriställchen. Schmp. $143^{\circ}$.

$\mathrm{C}_{22} \mathrm{H}_{24} \mathrm{~N}_{3}$ J. Ber.: C 57,76, H 5,25, N 9,19.

Gef.: C 55,78, H 5,36, N 9,03.

XI. 1 - Phenyl-2-methyl-3.5-bis - dimet hyla minophenyldivinylen-pyrazolium jo d i d

Analog I aus $1 \mathrm{~g} 1-\mathrm{Ph}$ enyl-2.3.5-trimethylp y a z o li u m jodid und 2 g p-Dimethyla m in o z i m t a ld e hy d. Dunkelrotbraune Kriställchen. Schmp. $188^{\circ}$.

$\mathrm{C}_{34} \mathrm{H}_{37} \mathrm{~N}_{3}$ J. Ber.: N 8,27. Gef.: N 8,37.

\title{
Zur Kenntnis der Triterpene
}

\section{Mitteilung ${ }^{1}$ : Synthese von 1-Isopropyl-4.5-dimethyl-indan}

\author{
Von L. Ruzicka, A. Brossi und O. Jeger \\ Aus dem Organisch-chemischen Laboratorium der Eidg. Technischen Hochschule Zürich \\ (Z. Naturforschg. 6 b, 246-249 [1951]; eingegangen am 11. Mai 1951) \\ John Eggert zum 60. Geburtstage gewidmet
}

Es wird die Synthese des bisher unbekannten 1-Isopropyl-4.5-dimethyl-indans (XI) beschrieben.

$\mathrm{F}$ ür Vergleichszwecke benötigten wir den im Titel angeführten Kohlenwasserstoff, der sich auf folgenden Wege herstellen ließ.

Der von Reichstein und Mitarbeitern ${ }^{2}$ beschriekene 2.3-Dimethyl-benzylalkohol (I) wurde durch Behandlung mit Bromwasserstoff in das Bromid(II) übergeführt, welches bei der Kondensation mit Malonsäure-dimethylester in Gegenwart von Natriummethylat den 2.3-Dimethyl-benzyl-malonester (III) lieferte. Bei der Verseifung des letzteren mit 10-proz.

1 161. Mitt.: Helv. chim. Acta 34, 1154 [1951].

2 T. Reichstein, A. Cohen, M. Ruth und H. F. M e ld a h l, Helv. chim. Acta 19, 415 [1936].

methanolischer Natronlauge entstand die in reiner Form nicht isolierte Dicarbonsäure IV, welche beim Erhitzen auf ungefähr $160^{\circ}$ Kohlendioxyd abspaltete und in die 2.3-Dimethyl-benzyl-essigsäure (V) überging. Aus $\mathrm{V}$ bereiteten wir mit Thionylchlorid das Säurechlorid VI, das nach Friedel-Crafts in Gegenwart von Aluminiumchlorid in guter Ausbeute zu dem bisher unbekannten 4.5-Dimethyl-indanon-(1) (VII) cyclisiert wurde. Die Verbindung VII weist im $\mathrm{UV}$ ein Absorptionsmaximum bei $260 \mathrm{~m} \mu, \log \varepsilon=4,1$ auf (Abb. A, Kurve I ${ }^{3}$ und wurde durch ein bei

3 Die in dieser Arbeit erwähnten UV-Absorptionsspektren wurden in alkoholischer Lösung aufgenommen. 
<smiles>[R]Cc1cccc(C)c1C</smiles>
$\mathrm{I}, \mathrm{R}=\mathrm{OH}$
II, $\mathrm{R}=\mathrm{Br}$<smiles>Cc1ccc2c(c1C)CC=C2C(C)C</smiles>

IX<smiles>Cc1ccc2c(c1C)CCC2C(C)C</smiles><smiles>Cc1cccc(CCC(C(=O)O)C(=O)O)c1C</smiles>

III, $\mathbf{R}=\mathrm{CH}_{3}$

IV, $\mathrm{R}=\mathrm{H}$<smiles>Cc1ccc2c(c1C)CCC2(O)C(C)C</smiles><smiles>Cc1ccc2c(c1C)CCC2=O</smiles>

VII

$\mathrm{V}, \mathrm{R}=\mathrm{OH}$

$\mathrm{VI}, \mathrm{R}=\mathrm{Cl}$<smiles>[3H]C</smiles><smiles>CC(C)=C1CCc2c(C)cccc21</smiles>

276-278 ${ }^{\circ}$ schmelzendes 2.4-Dinitro-phenylhydrazon sowie durch das in der Abb. B, Kurve 1, abgebildete IR-Absorptionsspektrum charakterisiert ${ }^{4}$.

Die Einwirkung von Isopropyl-magnesiumjodid auf das Keton VII führte zum tert. Carbinol VIII, welches bei der Destillation im Hochvakuum Wasser abspaltete und in einen Kohlenwasserstoff $\mathrm{C}_{14} \mathrm{H}_{18}$ überging. In diesem liegt wohl ein Gemisch der Isomeren der Struktur IX und $\mathrm{X}$ vor (UV-Absorptionsspektrum Abb. A, Kurve 2, IR-Absorptionsspektrum Abb. B, Kurve 2). Bei der katalytischen Hydrierung dieses Gemisches erhielten wir das gesuchte 1-Isopropyl-4.5dimethyl-indan (XI), welches bei $145-148^{\circ} / 11 \mathrm{~mm}$ siedet. Im UV und IR weist diese Verbindung die in der Abb. A, Kurve 3, bzw. Abb. B, Kurve 3, abgebildeten Absorptionsspektra auf.

Der Rockefeller Foundation in New York danken wir für die Unterstützung dieser Arbeit.

\section{Experimenteller Teil 5 \\ (Mitbearbeitet von R. G a u t s c h i) 2.3 - D i met hy l-benzy l bromid (II)}

17,9 g 2.3-Dimethyl-benzylalkohol (I)2 wurden in $50 \mathrm{~cm}^{3}$ Äther gelöst und durch die Lösung während $10 \mathrm{Stdn}$.

4 Herrn Dr. Hs. H. G ü $\mathrm{nth}$ a $\mathrm{rd}$ danken wir für die Aufnahme der IR-Absorptionsspektren, die auf einem B a i r d - „double-beam“-Spektrographen gemessen wurden.

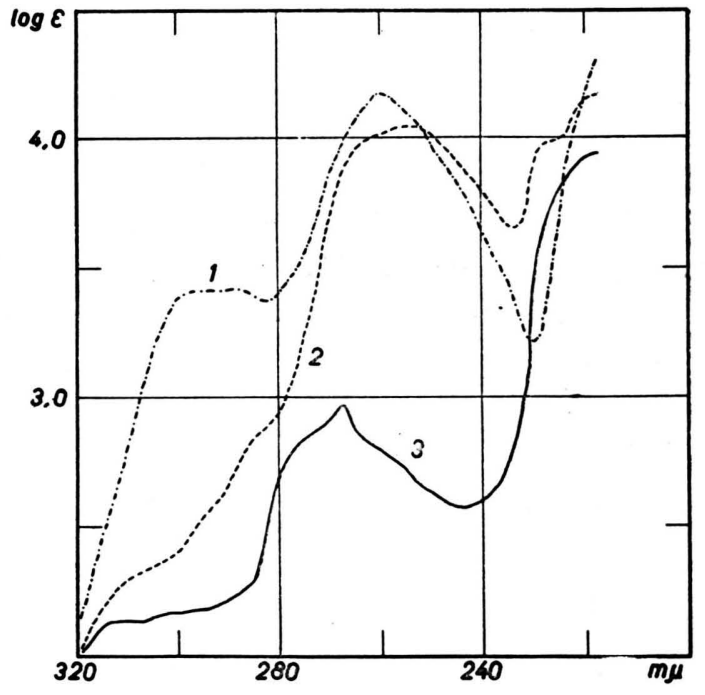

Abb. A. UV-Absorptionsspektren.

Kurve 1: 4.5-Dimethyl-indanon-(1)(VII),

Kurve 2: Gemisch der Kohlenwasserstoffe $\mathrm{C}_{14} \mathrm{H}_{18}$ (IX und $\mathrm{X}$ ),

Kurve 3: 1-Isopropyl-4.5-dimethyl-indan (XI).

unter Eiskühlung trockenes Bromwasserstoffgas durchgeleitet. Nach Eingießen in Eiswasser und Aufarbeiten wurden 25,6 g Rohprodukt erhalten, das bei der Destillation im Vig re u x - Kolben 20,5 g reines Bromid vom

5 Die Schmelzpunkte sind korrigiert und wurden in einer im Hochvakuum evakuierten Kapillare bestimmt. 

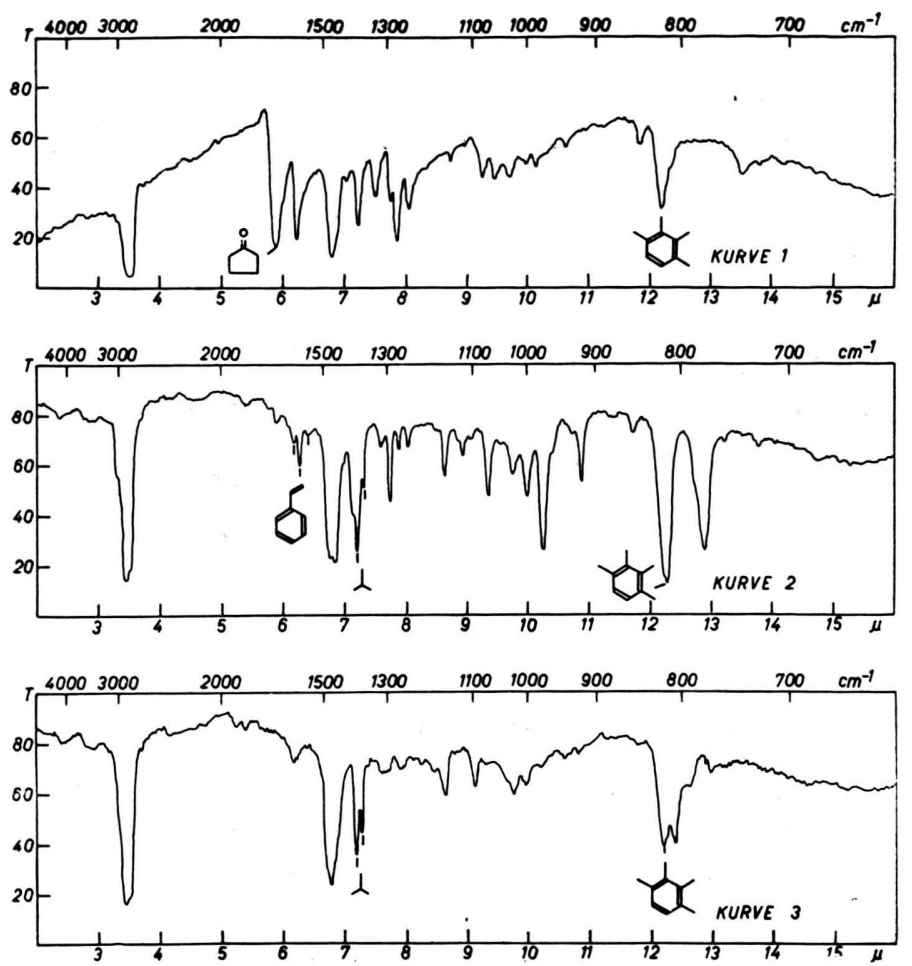

Abb. B. IR-Absorptionsspektren.

Kurve 1: 4.5-Dimethyl-indanon-(1) (VII),

Kurve 2: Gemisch der Kohlenwasserstoffe $\mathrm{C}_{14} \mathrm{H}_{18}$ (IX und X),

Kurve 3: 1-Isopropyl-4.5-dimethyl-indan (XI).

Sdp. ${ }_{11} 109-113^{\circ}$ lieferte. Eine Probe davon wurde zur Analyse nochmals destilliert; $n_{\mathrm{D}}^{20}: 1,575$.

3,726 mg Sbst. : 7,409 mg CO$, 1,895 \mathrm{mg}_{2} \mathrm{O}$.

$6,250 \mathrm{mg}$ Sbst. gaben $5,872 \mathrm{mg} \mathrm{AgBr}$.

$\mathrm{C}_{9} \mathrm{H}_{11}$ Br. Ber.: C 54,29, H 5,57, Br 40,14.

Gef.: C 54,27, H 5,69, Br 39,98.

Es liegt Verbindung II vor.

\section{3 - Dimethyl-benzyl-malonsäure - dimethylester (III)}

20 g Malonsäure-dimethylester ließ man unter Rühren zu einer Lösung von $2,5 \mathrm{~g}$ Natrium in $25 \mathrm{~cm}^{3}$ absol. Methanol zutropfen. Unter weiterem Rühren und Kühlen mit Eis wurden nun langsam $17 \mathrm{~g}$ 2.3-Dimethyl-benzylbromid (II) zugegeben und das Gemisch über Nacht bei Zimmertemperatur stehen gelassen. Nach der üblichen Aufarbeitung wurden $20 \mathrm{~g}$ kristallisierte, neutrale Produkte erhalten, die zur Analyse mehrmals aus Methanol umkristallisiert und bei Zimmertemperatur 48 Stdn. am Hochvakuum getrocknet wurden. Schmp. 70-71 .

$3,624 \mathrm{mg}$ Sbst.: $8,895 \mathrm{mg} \mathrm{CO}, 2,382 \mathrm{mg} \mathrm{H}_{2} \mathrm{O}$.

$4,004 \mathrm{mg}$ Sbst. verbr. bei der Methoxylbestimmung nach Vieböck u. B recher $9,663 \mathrm{~cm}^{3} 0,02-n$. $\mathrm{Na}_{2} \mathrm{~S}_{2} \mathrm{O}_{3}$.

$\mathrm{C}_{14} \mathrm{H}_{18} \mathrm{O}_{4}$. Ber.: C 67,18, H 7,25, $2 \mathrm{OCH}_{3} 24,80$. Gef.: C $66,98, \mathrm{H} 7,36,2 \mathrm{OCH}_{3} 24,97$.

Es liegt Verbindung III vor.

\section{3-Dimethyl-benzyl-essigsäure (V)}

$19 \mathrm{~g}$ Diester III wurden mit $110 \mathrm{~cm}^{3} 10$-proz. methanolischer Natronlauge 4 Stdn. unter Rückfluß gekocht und die Reaktionsprodukte in Äther aufgenommen. Durch Extrahieren mit $2-n$. Sodalösung gewann man daraus $15,5 \mathrm{~g} \mathrm{kri-}$ stalline, saure Produkte - wohl IV. Zur Decarboxylierung wurden diese im $\mathrm{H}$ i c k m a n n Kolben 1 Stde. auf $160^{\circ}$ erhitzt und hierauf im Hochvakuum destilliert. Die von $122-132^{\circ}$ siedende Fraktion kristallisierte nach einigem Stehenlassen. Durch Umkristallisieren aus Methanol erhielt man Prismen vom Schmp. 113 $-114^{\circ}$. Das Analysenpräparat wurde im Hochvakuum bei $90^{\circ}$ Blocktemperatur sublimiert.

3,624 mg Sbst.: $9,853 \mathrm{mg} \mathrm{CO} 2,2,566 \mathrm{mg} \mathrm{H}_{2} \mathrm{O}$.

$\mathrm{C}_{11} \mathrm{H}_{14} \mathrm{O}_{2}$. Ber.:

C 74,13, H 7,92. Gef.: C 74,22, H 7,92.

4.5 - D i m e th y l - ind a n o n - (1) (VII)

$7,5 \mathrm{~g}$ der Säure $V$ wurden mit $12 \mathrm{~cm}^{3}$ Thionylchlorid 2 Stdn. auf dem Dampfbad erhitzt. Das überschüssige Thionylchlorid wurde am Wasserstrahlvakuum entfernt; das rohe Säurechlorid VI unter starkem Rühren zu einer Suspension von $10 \mathrm{~g}$ Aluminiumchlorid in Petroläther so zugetropft, daß dieser durch die Reaktionswärme im Sieden gehalten wurde. Hierauf ließ man 2 Stdn. stehen und goß dann das dunkelrote Reaktionsprodukt auf Eis. Der mit verd. Sodalösung neutral gewaschene Ätherauszug wurde mit Tierkohle kurze Zeit gekocht und das nach dem Filtrieren und Abdampfen des Äthers gewonnene Keton aus Äther-Petroläther umkristallisiert. Man erhielt so 4,4 g Kristalle vom Schmp. 98-99 ${ }^{\circ}$. Das Analysenpräparat wurde $48 \mathrm{Stdn}$. bei Zimmertemperatur am Hochvakuum getrocknet.

$3,778 \mathrm{mg}$ Sbst. : $11,414 \mathrm{mg} \mathrm{CO}, 2,580 \mathrm{mg} \mathrm{H}_{2} \mathrm{O}$.

$\mathrm{C}_{11} \mathrm{H}_{12} \mathrm{O}$. Ber.: C 82,46, H 7,55. Gef.: C 82,45, H 7,64.

Es liegt Verbindung VI vor.

Das in üblicher Weise hergestellte 2.4-Dinitro-phenylhydrazon wurde aus Chloroform-Methanol mehrmals umkristallisiert. Das Analysenpräparat wurde 24 Stdn. bei $80^{\circ}$ am Hochvakuum getrocknet. Schmp. 276-278 ${ }^{\circ}$.

3,700 mg Sbst.: 8,092 $\mathrm{mg} \mathrm{CO}_{2}, 1,697 \mathrm{mg} \mathrm{H}_{2} \mathrm{O}$.

2,760 mg Sbst.: $0,409 \mathrm{~cm}^{3} \mathrm{~N}_{2}\left(18^{0}, 708 \mathrm{~mm}\right)$.

$\mathrm{C}_{17} \mathrm{H}_{18} \mathrm{O}_{4} \mathrm{~N}_{4}$. Ber.: C 59,64, H 5,30, N 16,37.

Gef.: C 59,68, H 5,13, N 16,20.

Überführung von VII in ein Gemisch der Kohlenwasserst of fe $\mathrm{C}_{14} \mathrm{H}_{18}$ (IX und X)

$1 \mathrm{~g}$ mit Jod aktiviertes Magnesium wurde mit $50 \mathrm{~cm}^{3}$ absol. Äther überschichtet, allmählich mit $8 \mathrm{~g}$ Isopropyljodid versetzt und zur Beendigung der Reaktion 1 Stde. auf dem Dampfbad erwärmt. Zu der so bereiteten Grignard-Verbindung gab man langsam eine Lösung von 4,0 g 
4.5-Dimethyl-indanon-(1) (VII) in $50 \mathrm{~cm}^{3}$ absol. Äther so $\mathrm{zu}, \mathrm{da}$ das Reaktionsgemisch in gelindem Sieden blieb. Es wurde nach der Zugabe noch 2 Stdn. auf dem Dampfbad erwärmt, worauf das Reaktionsgemisch mit kalter, gesättigter Ammoniumchloridlösung versetzt und in Äther aufgenommen wurde. Der nach dem Neutralwaschen und Abdampfen des Äthers verbleibende Rückstand (4,0 g) wurde im $\mathrm{H}$ ickman-Kolben im Hochvakuum destilliert und dabei $1,3 \mathrm{~g}$ einer Mittelfraktion vom Sdp. 72-82 abgetrennt. Diese wurde an $40 \mathrm{~g}$ Aluminiumoxyd (Akt. I/II) chromatographiert, wobei die ersten $200 \mathrm{~cm}^{3}$ Petroläther 1,0 g Substanz eluierten, die eine braune Farbreaktion mit Tetranitromethan gab. Das Analysenpräparat wurde im Wasserstrahlvakuum bei einer Badtemperatur von 152 bis $155^{\circ}$ über Natrium destilliert; $n_{\mathrm{D}}^{20}: 1,548$.

3,580 mg Sbst.: 11,842 mg $\mathrm{CO}_{2}, 3,132 \mathrm{mg} \mathrm{H}_{2} \mathrm{O}$. $\mathrm{C}_{14} \mathrm{H}_{18}$. Ber.: C 90,26, H 9,74. Gef.: C 90,27, H 9,79.

Es liegt ein Gemisch der Isomeren IX und $\mathrm{X}$ vor.
1 - I s o propyl-4.5 - d i m e th y l-ind a n (XI)

$380 \mathrm{mg}$ des Gemisches der Kohlenwasserstoffe $\mathrm{C}_{14} \mathrm{H}_{18}$ (IX und $\mathrm{X}$ ) wurden in $10 \mathrm{~cm}^{3}$ Eisessig gelöst und mit $50 \mathrm{mg}$ vorhydriertem Platinoxyd-Katalysator hydriert. Dabei wurden $45 \mathrm{~cm}^{3}$ Wasserstoff aufgenommen. Nach Eingießen in Wasser nahm man in Äther auf und erhielt nach Waschen mit 2-n. Natronlauge $350 \mathrm{mg}$ Substanz, welche zur Reinigung in Petroläther gelöst und durch eine Säule aus Aluminiumoxyd (Akt. I/II) filtriert wurde. Das nach dem Wegdampfen des Lösungsmittels gewonnene Produkt gab eine intensiv gelbe Tetranitromethanreaktion und wurde zur Analyse im Wasserstrahlvakuum über Natrium destilliert. $\mathrm{Sdp}_{11} 145-148^{\circ}$ (Badtemperatur); $n_{\mathrm{D}}^{19}: 1,522$.

3,346 mg Sbst.: 10,943 mg $\mathrm{CO}_{2}, 3,225 \mathrm{mg} \mathrm{H}_{2} \mathrm{O}$.

$\mathrm{C}_{14} \mathrm{H}_{20}$. Ber.: C 89,29, H 10,71. Gef.: C 89,25, H 10,79. Es liegt XI vor.

Die Analysen wurden in unserem mikroanalytischen Laboratorium von Hrn. W. M a n s e r ausgeführt.

\title{
Über die Herstellung von photographischen Kunststoffemulsionen
}

\author{
Von Albert Narath
}

Aus dem Institut für angewandte Photochemie der Techn. Universität Berlin-Charlottenburg

(Z. Naturforschg. 6 b, 249-251 [1951]; eingegangen am 18. Mai 1951)

\section{John Eggert zum 60. Geburtstage gewidmet}

\begin{abstract}
Es werden die Gesichtspunkte, die für die Herstellung von photographischen Kunststoffemulsionen maßgebend sind, erörtert. Auf Grund von Versuchsergebnissen wird ein Emulsionsrezept angegeben, nach welchem man Kunststoffemulsionen auf Polyvinylalkoholbasis herstellen kann. Die photographischen Eigenschaften einer derartigen Emulsion werden mitgeteilt.
\end{abstract}

$\mathrm{T}_{\mathrm{r}}$ rotz der hohen Leistungsfähigkeit der modernen photographischen Emulsionen hat es nicht an Versuchen gefehlt, die Gelatine als Einbettungsmittel für die lichtempfindlichen Silbersalze durch einen Kunststoff zu ersetzen. Der wesentlichste Nachteil der Gelatine besteht in der ungleichmäßigen und nicht voraussehbaren Qualität, die von der jeweiligen Beschaffenheit der verwendeten tierischen Ausgangsprodukte abhängt und erst nach ihrer Herstellung durch Ansatz von Versuchsemulsionen geprüft und beurteilt werden kann. Auch ein neues von A m mannBrass ${ }^{1}$ ausgearbeitetes Verfahren, das die Lichtstreuung zur Beurteilung der Gelatine heranzieht, setzt die Herstellung von Versuchsemulsionen mit der zu untersuchenden Gelatine voraus. Als rein physikalisches Verfahren vermeidet es zwar die Prüfung auf Grund entwickelter Emulsionsproben, gestattet

1 H. Ammann-Brass, Sci. Ind. photogr. 19, $401-418$ [1948]. aber natürlich auch nur die Aussage, ob und inwieweit das vorliegende Produkt für die Herstellung bestimmiter Emulsionen geeignet ist. Weitere Nachteile der Gelatine sind ihre Neigung, besonders bei hoher Luftfeuchtigkeit, zu schimmeln und durch Bakterienund Insektenfraß zerstört zu werden. Außerdem erfordert sie wegen ihres niedrigen Schmelzpunktes gegebenenfalls den Zusatz von Härtemitteln. Die Verwendung von Kunststoffen würde den wesentlichen Vorteil mit sich bringen, daß diese und die mit ihnen hergestellten Emulsionen in stets gleichmäßiger Qualität herstellbar sind, infolge höherer Schmelzpunkte keiner Härtungsmittel bedürfen und unempfindlich gegen Bakterien- und Insektenfraß sind.

Unter Berücksichtigung der genannten Tatsachen muß es Wunder nehmen, daß die Gelatine nicht schon längst durch Kunststoffe ersetzt worden ist. Der Grund hierfür ist vor allem darin zu suchen, daß die Gelatine ein vorzügliches Schutzkolloid darstellt und höchstempfindliche Emulsionen liefert. Erst neuer- 